\title{
Constraints Mitigation in Cognitive Radio Networks Using Cloud Computing
}

\author{
Dr. Bindhu V, \\ Professor and Head, Department of Electronics and Communication Engineering, \\ PPG Institute of Technology, \\ Villankurichi Saravanampatti P.O, \\ Coimbatore, Tamil Nadu, India.
}

\begin{abstract}
One of the most supportive technologies in enhancing the bandwidth utilization of the next generation network is cognitive radio network (CR-N). However the traditional CR-N is substantially constrained in accessing and the spectrum sensing, due to its limited, processing power and the storage capabilities. To advance the spectrum sensing performance and the spectrum management along with the development in the radio frequency resource allocation in the CR-N the paper clouts the cloud computing services in the proposed method to mitigate the constraints in the cognitive radio networking and also address the intrinsic security threats that are caused by the jamming in the CR-N. The performance of the proposed method is validated and the results are observed to evince the performance enhancement gained in managing the constraint in the CR-N using the cloud.
\end{abstract}

Keywords: Cloud Computing, Vast Storage, Computational Capability, Cognitive Radio Network, Spectrum Sensing, Spectrum Management

\section{Introduction}

The radio spectrum of the wireless networks is actually controlled by the policies with the fixed assignments enforced by the governmental agencies and allocated to the license holders on a long term basis. Despite the effective controlling implemented by the policies are in effect over the decades there is a continuous increase in the bandwidth request due to the propagation of ubiquitous internet and telecommunication services that were offered and requested by the mobile devices. The federal communication commission states that the utilization of the spectrum in the radio communication is not uniform and a quiet large portion remains unused. Many article based on the traditional wireless radio spectrum brings the fact that the spectrum for the geographical and the temporal allocation varies from $15.2 \%$ to $85.4 \%$ with the large area of spectrum attributes by a replacements and intermediate utilization whereas smaller areas, characterized by heavy spectrum usage. Consequently the limited spectrum availability and the inadequacy in the spectrum utilization increases the necessity of the communication, opportunistically exploiting the wireless

ISSN: $2582-4104$ 
Journal of trends in Computer Science and Smart technology (TCSST) (2020)

Vol.02/ No. 01

Pages: $1-14$

https://www.irojournals.com/tcsst/

DOI: https://doi.org/10.36548/jtcsst.2020.1.001

spectrum by dynamically adapting the communication configurations, that includes the transmission waveform, spectrum usage, channel accessing method, or the protocols used in networking.

The Cognitive radio network is one such enabling technology developed for the next generation communication and the networking. The CR-N enhances and further optimizes the radio electromagnetic spectrum integrating the cognitive capabilities that are achieved by uniting the analysis and the prediction of the radio scenes and the channel state estimation respectively and the reconfiguration capabilities achieved by the software defined radio upon which the cognitive radio is overlaid. Tasks of the cognitive holds both the machine learning and the signal processing techniques to perform a real time valuation of the radio environment interferences and evaluates the radio channels prevailing and the future states.

The users in the CR-N are categorized into two different kinds as primary users and secondary users, where the former is the radio spectrum license holder and the latter is the users who utilize the unused license spectrum temporarily.

The spectrum is directly utilized by the primary licensed user, meanwhile the unlicensed secondary users continuously monitors the availability in the spectrum and uses the portions of the spectrum that are unused without interfering the primary users. The figure. 1 below shows the how the spectrum is utilized by the CR-N.

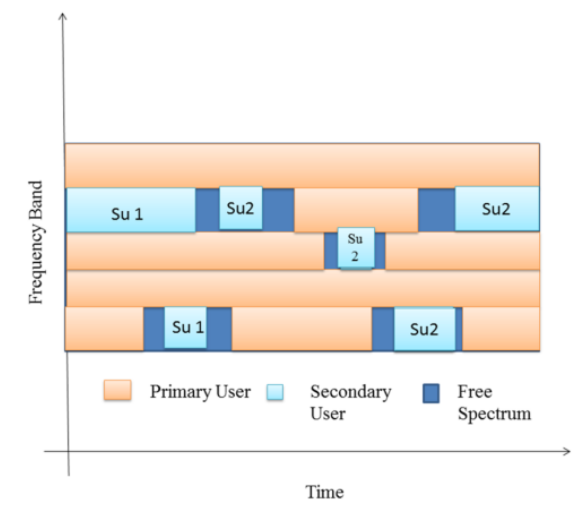

Fig .1.CR-N Spectrum Utilization

ISSN: $2582-4104$ 
Journal of trends in Computer Science and Smart technology (TCSST) (2020)

Vol.02/ No. 01

Pages: $1-14$

https://www.irojournals.com/tcsst/

DOI: https://doi.org/10.36548/jtcsst.2020.1.001

In order to improve the spectrum sensing and the spectrum management, the proposed method leverages the cloud computing that has a vast storage and the communication capabilities the proposed method in the paper is organized with the related works in section 2, proposed method in the section 3 performance improvement in section 4 and conclusion in section 5.

\section{Related Works}

Xiao et al [1] the author classifies the salient features of the wireless mesh architectures and the centralized architectures to leveraged from the view point of spectrum sensing in the cognitive radio networks, the book presents the complete particulars related to the modelling of the physical layer, medium access layer, routing layer and the cross layer considerations of the cognitive radio networks. Wang et al [2] presents the "survey on the recent advances related to the cognitive radios. The fundamental of cognitive radio technology, architecture and its applications, the paper reviews the prevailing spectrum sensing, important issue in dynamic spectrum allocation and sharing are investigated in the paper" the figure .2 presents the cognitive cycle.

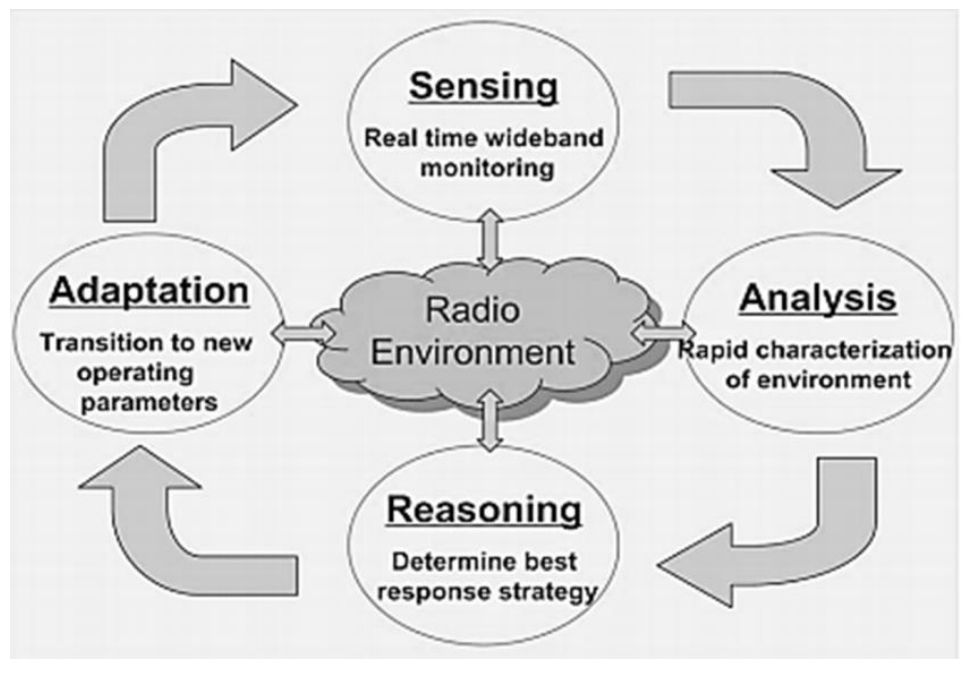

Fig.2 Cognitive Cycles [2]

Lee et al [3] the author in his paper has "developed an optimal spectrum sensing frame work based on the adaptive and co-operative sensing to optimize the sensing parameters in such a way to maximize the sensing efficiency and exploit multiple spectrum bands, spectrum selection and scheduling methods" Nie, et al [4]

ISSN: 2582-4104 
Journal of trends in Computer Science and Smart technology (TCSST) (2020)

Vol.02/ No. 01

Pages: $1-14$

https://www.irojournals.com/tcsst/

DOI: https://doi.org/10.36548/jtcsst.2020.1.001

the author in his paper utilizes the "game theory architecture for the examine the behavior of the cognitive radios for the distributed adaptive channels and utilizes the co-operative based spectrum sharing and finds that method to be enhance the overall network performance" Akyildiz, et al [5] This paper surveys the issues of co-operation method and how it is addressed using the co-operative sensing by examining the fundamental components of the co-operative sensing and also issues related in the wide band sensing. Quan, et al [6] the author utilizes the heuristic approaches and develops optimal linear co-operation architecture in spectrum sensing to identify accurately the weak primary signals. Zhang, et al [7] the author has proposed a "fast spectrum sensing algorithm for a large network which requires fewer cognitive radios to perform cooperative spectrum sensing while satisfying a given error bound" Valanarasu, Mr R., et al [8] is the survey on the wireless cognitive networks empowering the fifth generation technology. Kumar, T. et al [9] presents the methods of allocating the resources and the quality of service enhancements for the IOT paradigm using the FOG. Raj, Jennifer $S$ et al [10] proposes the "Virtual Structure for Sustainable Wireless Networks in Cloud Services and Enterprise Information System." Bhalaji, et al [11] proposes the "Delay Diminished Efficient Task Scheduling and Allocation for Heterogeneous Cloud Environment" Shakya, et al [12] proposes a "SSL and the migration tickets with the minimum privilege and does data encryption using the prediction based encryption. Darney, et al [13] "Performance Enhancements of Cognitive Radio Networks Using the Improved Fuzzy Logic." Ghasemi et al [14] is the survey on the requirements challenges and design tradeoffs in spectrum sensing" Akyildiz, et al [15] presents the survey on the "Next generation/dynamic spectrum access/cognitive radio wireless networks"

\subsection{Existing Methodology}

The cognitive radio being an intelligent technology is swiftly and unconventionally adaptable to the always changing conditions, working parameters and the environment; to rapidly identify the spectrum holes in the CR-N the spectrum sensing is done. The spectrum sensing is the fundamental component in the communications process of the cognitive radio's it is usually done prior to allocation of free spectrums to the spectrum sensing in the conventional CR-N's were considered as a hypothesis,-testing problem. The process of spectrum sensing monitors the spectrum of the primary users and identifies the free space in it and allocates it to the secondary users, once the primary person starts using his spectrum the Secondary user is dropped.

The free space identification probability was increased employing the detection techniques based on signals and the energy detection was one of the most reliable as it identified the free space, measuring the received signal strength for a fixed bandwidth for a certain time period $\left(P_{T}\right)$ for the cognitive radios

$\left(\mathrm{Cr}_{1}, \mathrm{Cr}_{2}, \ldots \ldots . \mathrm{Cr} r_{i}\right)$ the probability of the detection (D), missed detection (MD) and the false detection (FD) is stated below in the equation (1), (2), (3)

ISSN: $2582-4104$ 
Journal of trends in Computer Science and Smart technology (TCSST) (2020)

Vol.02/ No. 01

Pages: $1-14$

https://www.irojournals.com/tcsst/

DOI: https://doi.org/10.36548/jtcsst.2020.1.001

$$
\operatorname{prob}(F D)=\operatorname{prob}\left(E_{\text {freq }}>\text { instaneous enethd } / \text { unused slots }\right)
$$

Where $E_{\text {freq }}$ is the energy gathered in frequency domain, instaneous enethd is the instantaneous energy threshold.

$$
\operatorname{prob}(D)=\operatorname{prob}\left(E_{\text {freq }}>\text { instaneous }_{\text {enethd }} / \text { used slots }\right)
$$

$$
\operatorname{Prob}(M D)=1-\operatorname{prob}(D)
$$

but to address hidden terminal problems the co-operative sensing was used and in order to satisfy the secondary users the potential cloud computing was put into action the proposed method utilizes the cooperative spectrum sensing in the cloud computing to identify the un used primary spectrum and employ's a spectrum lessor to identify the proper unused spectrum band to the second user based on their requirement to avoid dropping of the secondary users when primary user comes into the scene. The vast storage capability and the computational capacity make the cloud cognitive radios to overcome its constraints on resource management and allocation.

\section{Proposed Methodology}

The evolving CR-N incorporating concepts like competition, spectrum sharing, heterogeneous radio networks and configurability requires ubiquitous and elastic computing infrastructures, that are equipped with the virtual unlimited resources to gather, examine, process and manage the radio communication events that are huge and dynamic. The spectrum whenever not in use by the primary users it is allotted to the secondary or unlicensed users who request for the spectrum, but many times the multitudes of secondary users often compete for the same spectrum, and this is impossible. So the cloud computing handles the spectrum allocation in such a way that the allocation benefits the Primary users and contents the quality of services requirements of the unlicensed users. The block diagram below in the fig. 3 shows the prototype of the Cloud based CR-N (CBCR-N).

ISSN: 2582-4104 
Journal of trends in Computer Science and Smart technology (TCSST) (2020)

Vol.02/ No. 01

Pages: $1-14$

https://www.irojournals.com/tcsst/

DOI: https://doi.org/10.36548/jtcsst.2020.1.001

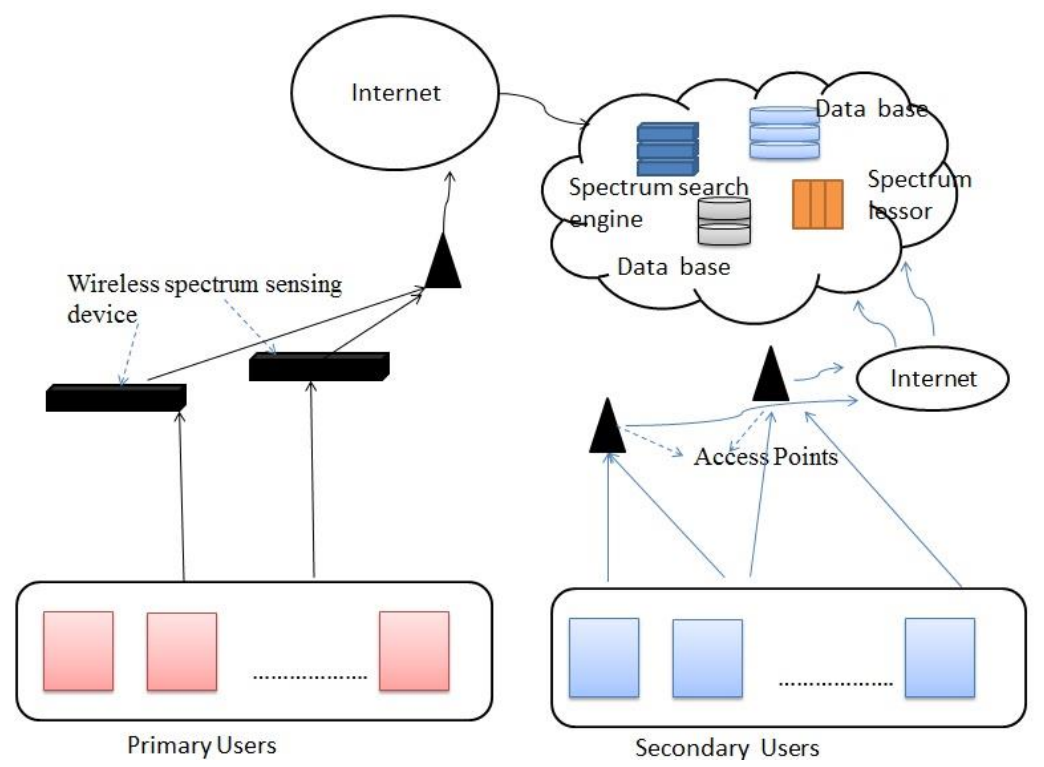

Fig.3 CBCR-N Prototype

The CBCR-N frame work is overlaid on the cloud and equipped with the distributed cognitive radio, the access point are interlinked to the cloud using the internet and the spectrum sensing is done employing the wireless spectrum sensing agents (SSA)/ devices that are connected to the access points. The SSA controls the cooperate spectrum sensing engine and receives an idea on the frequency map of the primary users and identifies the free space in its spectrum and reports to the cloud using the internet along with the duration of the free spectrum. The construction of the licensed user utilization map based on the sensed details is done using the sparse Bayesian algorithm. The step below shows the phases in the co-operating spectrum sensing in order to detect the free space in the frequency [7].

ISSN: $2582-4104$ 
Journal of trends in Computer Science and Smart technology (TCSST) (2020)

Vol.02/ No. 01

Pages: $1-14$

https://www.irojournals.com/tcsst/

DOI: https://doi.org/10.36548/jtcsst.2020.1.001

Every Cognitive Radio $\left(\mathrm{Cr}_{1}, \mathrm{Cr}_{2}, \ldots \ldots \mathrm{Cr} r_{i}\right)$

does

- Local spectrum measurement independently to make a binary decision $\{B D \in(0,1)\}$ for all $\mathrm{Cr}$.

- Forwards binary decisions to the public receiver either an access point or the Base station in case of the WLAN or mobile phone networks respectively.

- The receiver combines the $\{B D \in(0,1)\}$ and develops a final decision using the logic rule in the equation (4)

- Identifies the used and the unused portions of the licensed user in the frequency map.

The equation (4) gives the logic rule to combine all the decision gathered from all the cognitive radios,

$$
l_{r}=\sum_{t=1}^{i} B D\left\{\begin{array}{lr}
\geq n, & \text { used } \\
<n, & \text { un used }
\end{array}\right\}
$$

The sensed are transmitted to the cloud and updated in the spectrum database holding the details of the free spectrum and the duration. Now whenever, the secondary users request for the spectrum the spectrum lessor at the cloud gathers the details of the secondary user such as the access duration, and the time stamp of the request. The requests received are prioritized and queued using the queuing model (M/M/1). On receiving the request from the unlicensed user the spectrum lessor in the cloud, reads the data base to find out the free spaces in the spectrum and allocates the free space according to the location and the requirement of the unlicensed client.

The request of the unlicensed user (ULU) is registered through the access point to the cloud and indexes it in the home database that maintains the list of ULU and then accomplishes an peer to peer communication among one ULU to ULU, The access points of the CR-N periodically monitors the Spectrum lessor and gains knowledge about the data channel availability to and recasts the channel to the ULU if it is necessary. The same procedure is followed whenever an ordinary internet client request to get connected to the ULU. The fig. 4 below shows the protocol for the resource allocation and management in the CBCR-N.

ISSN: $2582-4104$ 
Journal of trends in Computer Science and Smart technology (TCSST) (2020)

Vol.02/ No. 01

Pages: $1-14$

https://www.irojournals.com/tcsst/

DOI: https://doi.org/10.36548/jtcsst.2020.1.001

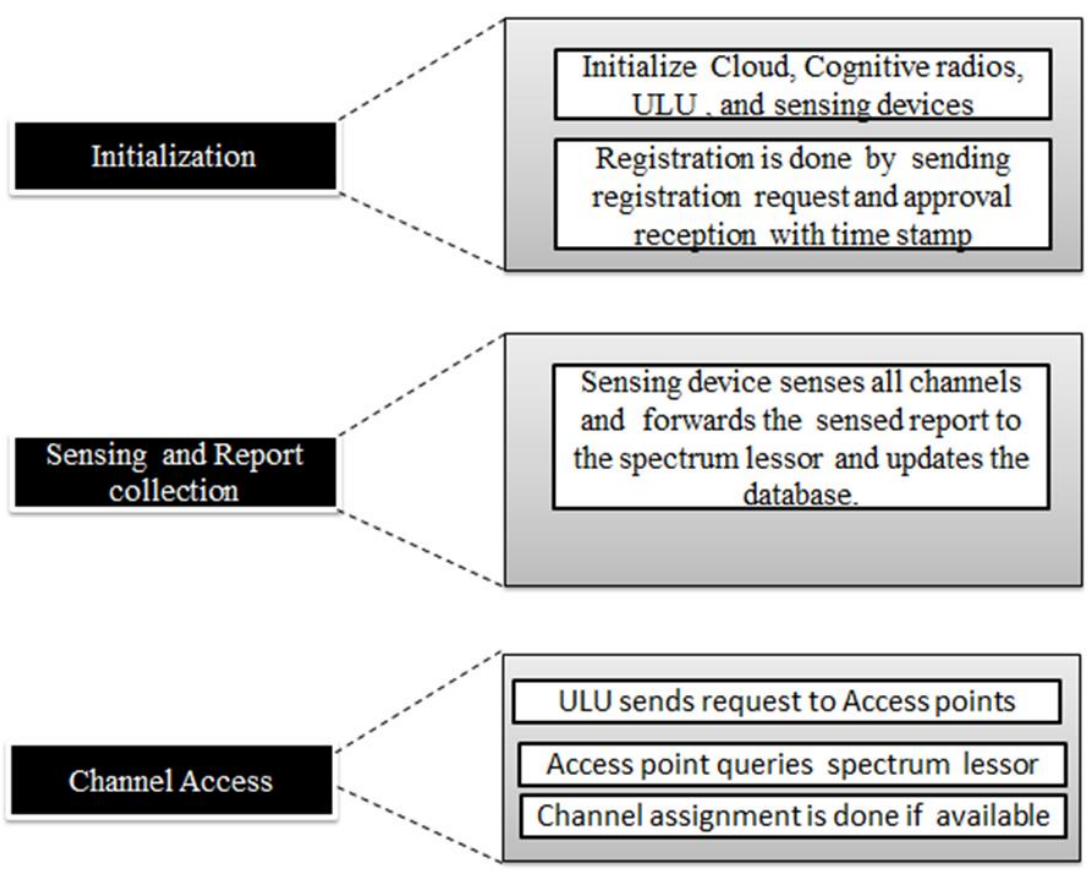

Fig.4 Resource Allocation Protocol

\section{Performance Evaluation}

The proposed CBCR-N with the cooperate sensing and the spectrum lessor is set up on the Amazon EC2 and the database is supported by the MySQL databases and simulated under different circumstances, such as (i) less weighed down, (ii) limited loads and (iii) more weighted down, the less weighed down circumstance are more preferable for the ULU as the competition among the ULU would be minimum. In the limited loads the free space are equal to the ULU and in more weighted down free space is low compared to ULU. For all the three circumstance the free space of the varying numbers of television, mobile phones and the satellites of are considered. The simulated results shows the sensing efficiency of the proposed method for the number of secondary users varying from 10 to 50 and percentage of satisfied ULU and the percentage of the benefits acquired by the LU form ULU, The table.1 below show the simulation parameters.

ISSN: $2582-4104$ 
Journal of trends in Computer Science and Smart technology (TCSST) (2020)

Vol.02/ No. 01

Pages: $1-14$

https://www.irojournals.com/tcsst/

DOI: https://doi.org/10.36548/jtcsst.2020.1.001

\begin{tabular}{|l|l|}
\hline \multicolumn{1}{|c|}{ Parameters } & \multicolumn{1}{c|}{ Values } \\
\hline Primary Users & $100-500$ \\
\hline Secondary users & $10-50$ \\
\hline Access point used & 10 per location \\
\hline Wireless sensing devices & $\begin{array}{l}100-150 \text { per } \\
\text { area }\end{array}$ \\
\hline $\begin{array}{l}\text { No. Of Television considered for \{(i), (ii), } \\
\text { (iii) }\}\end{array}$ & $20,33,40$ \\
\hline $\begin{array}{l}\text { No. of satellites considered for \{(i), (ii), } \\
\text { (iii) }\}\end{array}$ & $5,10,33$ \\
\hline $\begin{array}{l}\text { No. of mobile phones considered for }\{\text { (i), } \\
\text { (ii), (iii) }\}\end{array}$ & $6,10,24$ \\
\hline
\end{tabular}

Table.1 simulation Parameters

The fig. 5 shows the percentage of the satisfied users under the three different cases, for varying number of ULU, totally $40 \mathrm{ULU}$ were acknowledged out of the $50 \mathrm{ULU}$ and only $18 \%$ were satisfied under the third circumstance, $45 \%$ were satisfied in the second circumstance and the totally $80 \%$ were satisfied in the first circumstance. 
Journal of trends in Computer Science and Smart technology (TCSST) (2020)

Vol.02/ No. 01

Pages: $1-14$

https://www.irojournals.com/tcsst/

DOI: https://doi.org/10.36548/jtcsst.2020.1.001

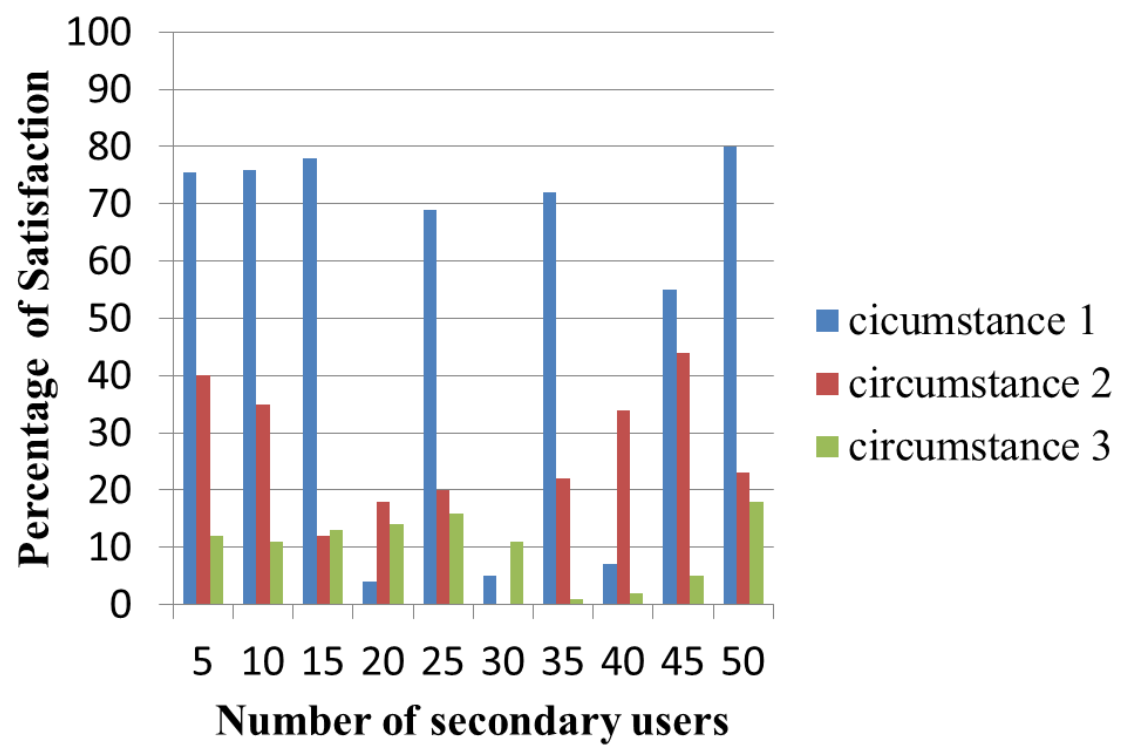

Fig.5 Percentage of Satisfaction

The fig. 6 shows the percentage of benefits enjoyed by the licensed user excluding the charges levied by the cloud in allocating the free space. The percentages of the benefits were observed for the 40 user admitted as the 10 users were not approved due to their heavy amount of data rate required. The benefits increased with the number of ULU, in circumstance three the benefits observed were zero as the channel access was denied. 
Journal of trends in Computer Science and Smart technology (TCSST) (2020)

Vol.02/ No. 01

Pages: $1-14$

https://www.irojournals.com/tcsst/

DOI: https://doi.org/10.36548/jtcsst.2020.1.001

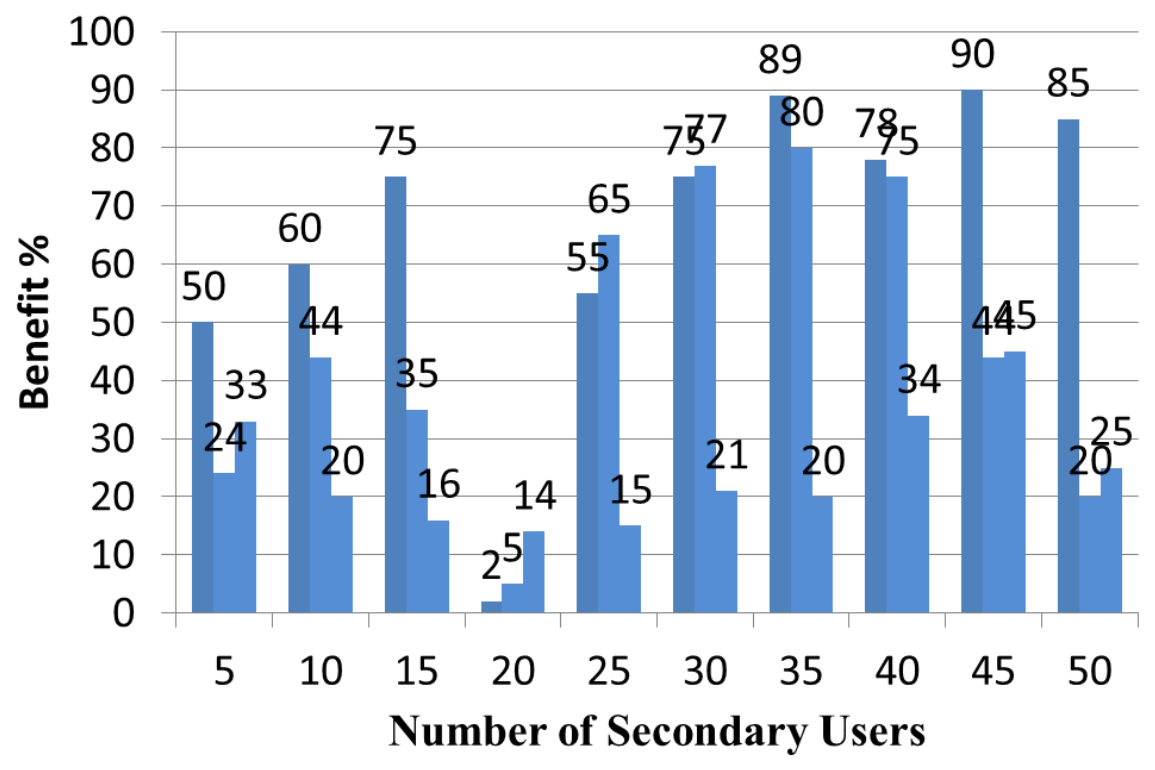

Fig.6 Benefits Percentage

The fig. 7 below shows the sensing efficiency of the proposed method over a certain observation time and number of licensed users. 
Journal of trends in Computer Science and Smart technology (TCSST) (2020)

Vol.02/ No. 01

Pages: $1-14$

https://www.irojournals.com/tcsst/

DOI: https://doi.org/10.36548/jtcsst.2020.1.001

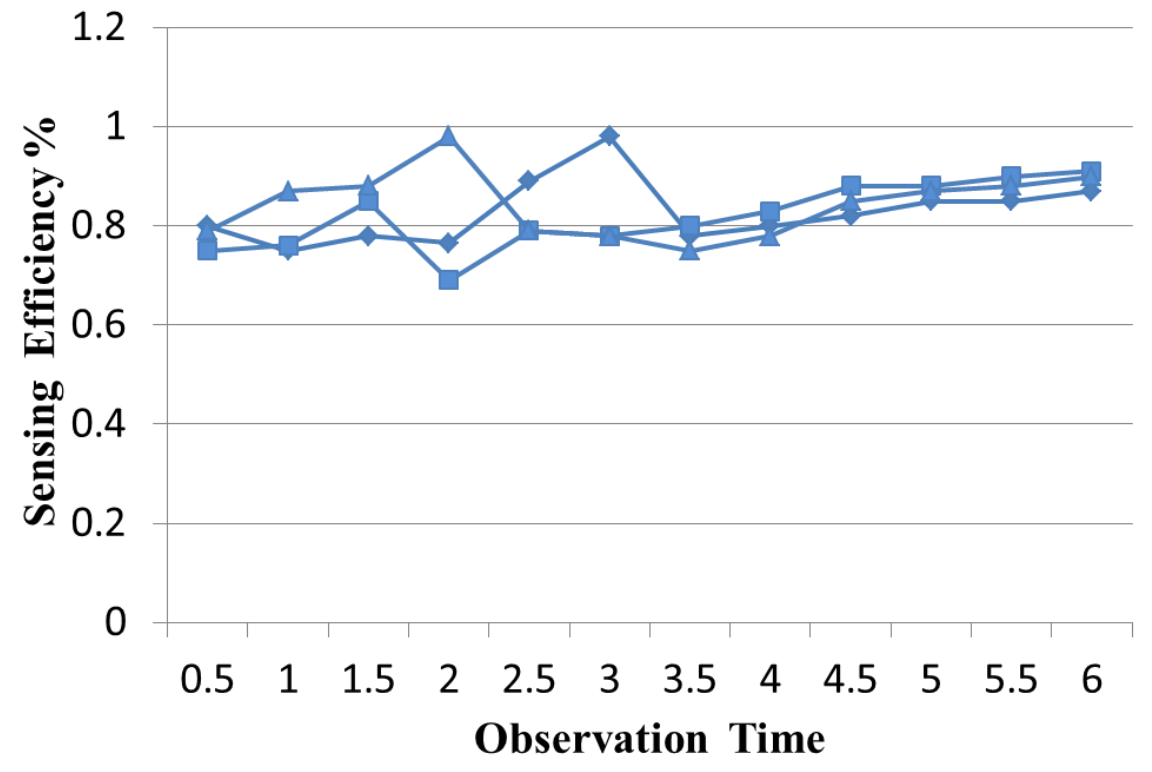

Fig. 7 Sensing Efficiency

The results observed over varying period, shows the sensing efficiency of the proposed method to further enhance the efficiency the paper is use the more advanced searching algorithms in the future.

\section{Conclusion}

With the development of the cloud- based cognitive radio networking the public would enjoy a seamless connection and a wireless access to the internet, so the proposed method utilizes the cloud to manage and allocate the free space of the licensed users to the ULU by monitoring the Spectrum utilizing the co-operate spectrum sensing and the sparse Bayesian algorithm to map the free space and convey it to the cloud data base and also uses a spectrum lessor to take care of the allocation of the free spaces to the ULU. The results observed under different circumstance validates the performance of the proposed method in terms of the sensing efficiency satisfied users and paybacks enjoyed by the licensed users. IN future the paper is to engage an advanced searching algorithm to sensing the free spaces in the LU minimizing the false and missed identification. 
Journal of trends in Computer Science and Smart technology (TCSST) (2020)

Vol.02/ No. 01

Pages: $1-14$

https://www.irojournals.com/tcsst/

DOI: https://doi.org/10.36548/jtcsst.2020.1.001

\section{References}

[1] Xiao, Yang, and Fei Hu, eds. Cognitive radio networks. CRC press, 2008.

[2] Wang, Beibei, and KJ Ray Liu. "Advances in cognitive radio networks: A survey." IEEE Journal of selected topics in signal processing 5, no. 1 (2010): 5-23.

[3] Lee, Won-Yeol, and Ian F. Akyildiz. "Optimal spectrum sensing framework for cognitive radio networks." IEEE Transactions on wireless communications 7, no. 10 (2008): 3845-3857.

[4] Nie, Nie, and Cristina Comaniciu. "Adaptive channel allocation spectrum etiquette for cognitive radio networks." Mobile networks and applications 11, no. 6 (2006): 779-797.

[5] Akyildiz, Ian F., Brandon F. Lo, and Ravikumar Balakrishnan. "Cooperative spectrum sensing in cognitive radio networks: A survey." Physical communication 4, no. 1 (2011): 40-62.

[6] Quan, Zhi, Shuguang Cui, and Ali H. Sayed. "Optimal linear cooperation for spectrum sensing in cognitive radio networks." IEEE Journal of selected topics in signal processing 2, no. 1 (2008): 28-40.

[7] Zhang, Wei, Ranjan K. Mallik, and Khaled Ben Letaief. "Cooperative spectrum sensing optimization in cognitive radio networks." In 2008 IEEE International Conference on Communications, pp. 3411-3415. IEEE, 2008.

[8] Valanarasu, Mr R., and A. Christy. "COMPREHENSIVE SURVEY OF WIRELESS COGNITIVE AND 5G NETWORKS." Journal of Ubiquitous Computing and Communication Technologies (UCCT) (2019): 23-32.

[9] Kumar, T. Senthil. "Efficient resource allocation and QOS enhancements of IoT with FOG network." J ISMAC 1 (2019): 101-110.

[10] Raj, Jennifer S., and S. Smys. "VIRTUAL STRUCTURE FOR SUSTAINABLE WIRELESS NETWORKS IN CLOUD SERVICES AND ENTERPRISE INFORMATION SYSTEM." Journal of ISMAC 1, no. 03 (2019): 188-204.

[11] Bhalaji, N. "DELAY DIMINISHED EFFICIENT TASK SCHEDULING AND ALLOCATION FOR HETEROGENEOUS CLOUD ENVIRONMENT." Journal of trends in Computer Science and Smart technology (TCSST) 1, no. 01 (2019): 51-62.

[12] Shakya, Subarna. "AN EFFICIENT SECURITY FRAMEWORK FOR DATA MIGRATION IN A CLOUD COMPUTING ENVIRONMENT." Journal of Artificial Intelligence 1, no. 01 (2019): 45-53.

[13] Darney, P. Ebby, and I. Jeena Jacob. "PERFORMANCE ENHANCEMENTS OF COGNITIVE RADIO NETWORKS USING THE IMPROVED FUZZY LOGIC." Journal of Soft Computing Paradigm (JSCP) 1, no. 02 (2019): 57-68.

[14] Ghasemi, Amir, and Elvino S. Sousa. "Spectrum sensing in cognitive radio networks: requirements, challenges and design trade-offs." IEEE Communications magazine 46, no. 4 (2008): 32-39.

ISSN: $2582-4104$ 
Journal of trends in Computer Science and Smart technology (TCSST) (2020)

Vol.02/ No. 01

Pages: $1-14$

https://www.irojournals.com/tcsst/

DOI: https://doi.org/10.36548/jtcsst.2020.1.001

[15] Akyildiz, Ian F., Won-Yeol Lee, Mehmet C. Vuran, and Shantidev Mohanty. "NeXt generation/dynamic spectrum access/cognitive radio wireless networks: A survey." Computer networks 50, no. 13 (2006): 2127-2159. 